\section{Nutritional intervention for}

\section{cancer sarcopenia}

\author{
Takuya Mori', Akira Kido², Isao Kawahara', Shota Nuaga', \\ Yoshihiro Miyagawa', Kei Goto', Shiori Mori', Shingo Kishi', \\ Kiyomu Fujii', Rina Fujiwara-Tani' and Hiroki Kuniyasu' ${ }^{1 *}$
}

1Department of Molecular Pathology, Nara Medical University, 840 Shijo-cho, Kashihara, Nara 6348521, Japan

${ }^{2}$ Department of Rehabilitation Medicine, Nara Medical University, 840 Shijo-cho, Kashihara, Nara 6348522, Japan
Received: 23 December 2020

Accepted: 05 January, 2021

Published: 06 January, 2021

*Corresponding authors: Hiroki Kuniyasu, Department of Molecular Pathology, Nara Medical University, 840 Shijo-cho, Kashihara, Nara, 634-8521, Japan, Tel: +81744-22-3051; Fax: +81-744-25-7308;

E-mail: cooninh@zb4.so-net.ne.jp

Keywords: Cancer sarcopenia; Cachexia; Mediumchain fatty acid

https://www.peertechz.com

Check for updates

\title{
Abstract
}

Most patients with advanced cancer develop skeletal muscle atrophy called sarcopenia, which reduces treatment tolerance and social activity and worsens the prognosis. Glucose suppresses skeletal muscle atrophy in cancer-bearing mice, while promoting cancer growth. In contrast, medium-chain fatty acids reduce skeletal muscle atrophy and suppress cancer growth. Simultaneous administration of glucose and medium-chain fatty acids suppresses skeletal muscle atrophy and eliminates the tumor growth seen in glucose. Based on these findings, dietary intervention using a combination of glucose and medium-chain fatty acids is expected to be effective in suppressing sarcopenia in cancer patients.

\section{Introduction}

Cachexia affects $80 \%$ of advanced-stage cancer patients and is a direct cause of death in $30 \%[1,2]$. As defined by the 2008 cachexia consensus conference "cachexia, is a complex metabolic syndrome associated with underlying illness and characterized by loss of muscle with or without loss of fat mass"[3], skeletal muscle atrophy is considered a major pathology of cancer cachexia [4,5]. In addition, decreased skeletal muscle mass in cancer patients is not only associated with decreased quality of life for patients, but is also closely associated with survival prognosis [3]. On the other hand, suppressing skeletal muscle atrophy significantly prolongs life prognosis regardless of tumor burden [6]. From these findings, improvement of skeletal muscle atrophy in cancer cachexia is an important issue. In recent years, exercise for improving malnutrition, anticancer treatment, and activity enhancement has been recommended in patients with cachexia [7]. In this review, we focus on nutritional interventions and show the possibility of nutritional interventions aimed at preventing skeletal muscle atrophy using an animal model of cancer cachexia.

\section{Cancer cachexia and nutritional intervention}

Cancer cachexia causes anorexia and weight loss in cancer patients, eventually leading to irreversible malnutrition [8]. Along with this, the skeletal muscles are atrophied, and the tolerance for treatment with anticancer agents. Treatment of cancer cachexia requires measures to manage reduced food intake and address catabolism as a result of inflammation [9]. Therefore, nutritional intervention has recently been proposed as a treatment for cancer patients with malnutrition $[10,11]$. However, nutrients such as carbohydrates and linoleic acid may promote tumor growth [12-14]. Therefore, nutrition interventions in cancerous sarcopenia require careful consideration of their effects on tumors as well as skeletal muscle. However, while there are many reports on the effects of nutritional interventions in cancerous sarcopenia, there are few reports on nutritional interventions that focus on both skeletal muscle and tumors.

\section{Effects of carbohydrates on tumors and skeletal muscle}

Glucose is an essential nutrient for skeletal muscle cells, leading to muscle contraction, homeostasis, and muscle cell growth [15]. Glucose uptake in skeletal muscle cells is insulin 
dependent via GLUT4 [16]. On the other hand, glucose is known to have a growth promoting action also in tumor cells, and it is known that the tumor cells selectively produce energy by glycolytic metabolism as a Warburg effect [17]. In addition, glucose uptake by tumor cells is insulin-independent by GLUT1, which has a higher sugar affinity, and it is thought that blood glucose easily promotes tumor growth [18]. Although tumor suppressive effects of carbohydrate restriction have been reported [19], it has been reported glucose restriction suppresses skeletal muscle differentiation and results in muscle atrophy [20]. Therefore, it is considered that the carbohydrate restriction on the tumor inhibits the energy production of skeletal muscle and exacerbates skeletal muscle atrophy. However, there are few reports that simultaneously examined the effects of carbohydrate loading on tumors and skeletal muscle in cancer-bearing bodies. Therefore, we investigated the effects of carbohydrate loading on tumors and skeletal muscle in a mouse tumor-bearing model [21]. We inoculated subcutaneously on the back of BALB/c mice with CT26 cells, a syngeneic mouse colon cancer cell line, and allowed them to freely drink sugar water ( $0 \% 10 \% 50 \%$ glucose) for 2 weeks to analyze tumors and skeletal muscle. As a result, the subcutaneous tumor diameter increased in a glucose concentration-dependent manner. In addition, a significant negative correlation was confirmed between the tumor diameter and blood glucose level. Muscle weight was significantly low in the tumor group; however, SDSsoluble myosin light chain 1 (SDS-MLC1), which indicates the functional maturity of skeletal muscle, showed a significantly high value in a glucose concentration-dependent manner. At $50 \%$ sugar concentration, improvement was observed to a level equivalent to that of the control group (non-cancer-bearing mice) [22]. From the above, it was clarified that the glucose load in the tumor-bearing body promotes the increase in tumors, while increasing the functional maturity of skeletal muscle and improving skeletal muscle atrophy. Therefore, it is necessary to carefully consider the administration of carbohydrates to cancer-bearing bodies.

\section{Effects of medium-chain fatty acids on tumors and ske- letal muscle}

Intake of medium-chain fatty acids has been shown to improve metabolic syndrome [22] and induction of apoptosis in cancer cells has also been reported [23]. We have also reported the antitumor effect of medium-chain fatty acids and ketones [24]. After fatty acid undergoes $\beta$-oxidation in mitochondria, the acetyl CoA produced is metabolized in the TCA cycle, and ATP is produced by oxidative phosphorylation. Since long-chain fatty acids are used for energy production, intracellular uptake by transporters such as CD36 and fatty acid binding proteins and translocation into the mitochondria by carnitine shuttle are required $[25,26]$. In contrast, Medium-chain fatty acids are taken into mitochondria without carnitine shuttle, undergo $\beta$-oxidation, and are used for oxidative phosphorylation in the TCA cycle [27]. Thus medium-chain fatty acids are rapidly utilized in mitochondria in equilibrium with changes in blood concentration [28]. Intake of medium-chain fatty acids is expected to forcefully promote mitochondrial metabolism in cells. Mitochondria have been reported to have dysfunction and poor quality control in tumor cells $[29,30]$, and we have found that forced metabolism induces excessive oxidative stress production and apoptosis [24]. On the other hand, medium chain fatty acids in skeletal muscle have been reported to lead to improvement of mitochondrial energy metabolism in skeletal muscle via GPR84 [31]. In addition, normal mitochondria can produce ATP much more efficiently than glycolysis, and thus may promote skeletal muscle growth.

Based on these findings, we investigated the effect of oral intake of medium-chain fatty acids on skeletal muscle hypertrophy in a mouse model [21]. A LAA diet containing lauric acid (LAA, C12: $0,0 \%, 2 \%, 5 \% \mathrm{w} / \mathrm{w}$ ) added to a control diet was orally ingested for 2 weeks. As a result, skeletal muscle wet weight was significantly increased in the $2 \%$ LAA diet, but significantly decreased in the 5\% LAA diet. From these results, it was confirmed that medium-chain fatty acids act to promote skeletal muscle growth at an appropriate concentration. On the other hand, it was shown that at high concentration, excessive mitochondrial activation also causes oxidative stress in skeletal muscle and induces muscle atrophy. Muscle atrophy due to excessive oxidative stress production by LAA is also observed in myocardium [32].

Based on these results, we used a $2 \%$ LAA diet, which is considered to be an appropriate concentration for the murine cachexia model, and orally ingested it for 2 weeks [21]. In our cachexia model, male BALB/c mice are inoculated intraperitoneally with syngeneic CT26 colon cancer cells, and cachexia phenotypes such as ascites retention, weight loss, and skeletal muscle atrophy are induced in about 10 days [21]. When a $2 \%$ LAA diet was orally administered to the cachexia group, an increase in skeletal muscle mass and an increase in skeletal muscle SDS-MLC1 were confirmed. On the other hand, in tumors, a $2 \%$ LAA diet resulted in a decrease in tumor weight and a decrease in ascites retention [21]. Thus, oral ingestion of medium-chain fatty acids is suggested to improve skeletal muscle atrophy and suppress tumors in cancer cachexia model, and medium-chain fatty acids are important in nutritional intervention for cancer sarcopenia. Was considered to be a nutrient.

\section{Effect of combined intake of medium-chain fatty acid and carbohydrate on tumor and skeletal muscle}

As described above, glucose and medium-chain fatty acids are the same energy source, but are metabolized by different pathways in intracellular metabolism. Therefore, simultaneous ingestion of both may improve skeletal muscle atrophy without promoting tumor growth. Therefore, in two types of mouse cachexia models of CT26 mouse colon cancer cells and HT29 human colon cancer cells, $10 \%$ glucose drinking water, $2 \%$ lauric acid diet alone, or the effect of the combined use, after oral ingestion for 2 weeks, The tumor and skeletal muscle were removed and examined [21]. As shown in Table 1, the weight of skeletal muscle decreased in the cachexia group, but significantly increased with the combined intake, and improved to the same level as the non-tumor bearing mice. In addition, there was no significant difference in tumor weight between the combination group and the control diet group.

Citation: Mori T, Kido A, Kawahara I, Nuaga S, Kuniyasu H, et al. (2021) Nutritional intervention for cancer sarcopenia. Ann Musculoskelet Med 5(1): 001-004. 
Table 1: Effect of dietary intervention using glucose and/or lauric acid on cancer sarcopenia.

\begin{tabular}{|c|c|c|c|c|}
\hline $\begin{array}{c}\text { Tumor cell } \\
\text { line }\end{array}$ & Diet & $\begin{array}{c}\text { Tumor weight } \\
(\mathbf{g})^{\mathbf{1}}\end{array}$ & $\begin{array}{c}\text { Muscle weight } \\
(\mathbf{g})^{2}\end{array}$ & $\begin{array}{c}\text { SDS-MLC } \\
(\mathbf{p g} / \mathbf{g})\end{array}$ \\
\hline CT26 & No tumor/control & - & $0.15 \pm 0.002$ & $23 \pm 1.3$ \\
\hline & Control & $1.35 \pm 0.15$ & $0.11 \pm 0.002$ & $13 \pm 1.2$ \\
\hline & LAA & $0.63 \pm 0.06$ & $0.13 \pm 0.01$ & $22 \pm 3.5$ \\
\hline & LAA+glucose & $1.58 \pm 0.16$ & $0.14 \pm 0.01$ & $27 \pm 3.1$ \\
\hline & No tumor/control & - & $0.15 \pm 0.01$ & $21 \pm 2.2$ \\
\hline & Control & $1.2 \pm 0.11$ & $0.09 \pm 0.002$ & $11 \pm 1.2$ \\
\hline & LAA & $0.3 \pm 0.04$ & $0.14 \pm 0.01$ & $21 \pm 2.7$ \\
\hline & LAA+glucose & $1.25 \pm 0.13$ & $0.15 \pm 0.01$ & $23 \pm 2.5$ \\
\hline
\end{tabular}

1) To measure tumor weight, the peritoneal tumors were dissected from the intestine, mesenterium, diaphragm, and abdominal wall, grossly removing nontumoral tissues.

2) The quadriceps femoris muscle was cut at the muscle end on the upper edge of the patella, peeled off from the femur, and separated at the muscle origin on the frontal surface of the anterior lower iliac spine. The excised quadriceps femoris muscle was weighed immediately, avoiding drying.

3) Whole cell lysate solubilized with $0.1 \%$ SDS-RIPA buffer from sonicated muscle tissue was analyzed with ELISA for myosin light chain $1 / 3$ isoform

Glucose is an important nutrient for the prevention of skeletal muscle atrophy; however, administration alone promoted tumor growth. In contrast, the simultaneous ingestion of lauric acid into glucose offsets the tumor-growing effect of carbohydrates in tumors by the antitumor effect of lauric acid, and the synergistic effect of carbohydrates and lauric acid on skeletal muscle suppressed sarcopenia. In nutritional intervention in cancer patients, combined intake of carbohydrates and medium-chain fatty acids was found to suppress skeletal muscle atrophy without causing tumor growth, and is useful for improving or preventing cancer sarcopenia.

\section{Conclusion}

Nutritional interventions in cancerous sarcopenia require simultaneous consideration of their effects on tumor growth and skeletal muscle atrophy. Co-administration of mediumchain fatty acids and glucose suppressed cancerous sarcopenia without increasing tumors. It is considered that the combined use of glucose and medium-chain fatty acids is effective for the prevention of cancerous sarcopenia. Future clinical applications are strongly expected.

\section{Funding sources}

This work was supported by MEXT KAKENHI Grant Number $20 \mathrm{~K} 11260$ (OH), 19K16564 (RFT), 19K19915 (KG), $20 \mathrm{~K} 18007$ (KS), $20 \mathrm{~K} 19349$ (IK) and 18K10788 (KF).

\section{Acknowledgment}

The authors thank Ms. Tomomi Masutani for expert assistance with the preparation of this manuscript.

\section{References}

1. Fearon KC (2008) Cancer cachexia: developing multimodal therapy for a multidimensional problem. Eur J Cancer 44: 1124-1132. Link: http://bit.ly/391jWMv
2. Blum D, Stene GB, Solheim TS, Fayers P, Hjermstad MJ, et al. (2014) Validation of the Consensus-Definition for Cancer Cachexia and evaluation of a classification model--a study based on data from an international multicentre project (EPCRC-CSA). Ann Oncol 25: 1635-1642. Link: http://bit.ly/2XaX8Ev

3. Evans WJ, Morley JE, Argiles J, Bales C, Baracos V, et al. (2008) Cachexia: A new definition. Clin Nutr 27: 793-799. Link: http://bit.ly/3b8TCCX

4. Acharyya S, Butchbach ME, Sahenk Z, Wang H, Saji M, et al. (2005) Dystrophin glycoprotein complex dysfunction: a regulatory link between muscular dystrophy and cancer cachexia. Cancer Cell 8: 421-432. Link: http://bit.ly/3nh411y

5. Argilés JM, Stemmler B, López-Soriano FJ, Busquets S (2015) Nonmuscle Tissues Contribution to Cancer Cachexia. Mediators Inflamm 2015: 182872. Link: http://bit.ly/38eL37x

6. Zhou X, Wang JL, Lu J, Song Y, Kwak KS, et al. (2010) Reversal of cancer cachexia and muscle wasting by ActRIIB antagonism leads to prolonged survival. Cell 142: 531-543. Link: http://bit.ly/390HkcT

7. Fearon K, Arends J, Baracos V (2013) Understanding the mechanisms and treatment options in cancer cachexia. Nat Rev Clin Oncol 10: 90-99. Link: http://bit.ly/3b9WuQ0

8. Ezeoke CC, Morley JE (2015) Pathophysiology of anorexia in the cancer cachexia syndrome. J Cachexia Sarcopenia Muscle 6: 287-302. Link: http://bit.ly/3ohpnNF

9. Argiles JM, Lopez-Soriano FJ, Stemmler B, Busquets S (2017) Novel targeted therapies for cancer cachexia. Biochem J 474: 2663-2678. Link: http://bit.ly/35dF2pY

10. Laviano A, Di Lazzaro Giraldi G, Koverech A (2016) Does nutrition support have a role in managing cancer cachexia? Curr Opin Support Palliat Care 10: 288292. Link: http://bit.ly/3pLRqVZ

11. Mantovani G, Madeddu C (2010) Cancer cachexia: medical management Support Care Cancer 18: 1-9. Link: http://bit.ly/3neP3sY

12. Shimomoto T, Ohmori H, Luo Y, Chihara Y, Denda A, et al. (2012) Diabetesassociated angiotensin activation enhances liver metastasis of colon cancer. Clin Exp Metastasis 29: 915-925. Link: http://bit.ly/3pPeXFA

13. Shimomoto $T$, Luo $Y$, Ohmori $H$, Chihara $Y$, Fujii $K$, et al. (2012) Advanced glycation end products (AGE) induce the receptor for AGE in the colonic mucosa of azoxymethane-injected Fischer 344 rats fed with a highlinoleic acid and high-glucose diet. J Gastroenterol 47: 1073-1083. Link: http://bit.ly/3nd5PZp

14. Ohmori H, Luo Y, Fujii K, Sasahira T, Shimomoto T, et al. (2010) Dietary linoleic acid and glucose enhances azoxymethane-induced colon cancer and metastases via the expression of high-mobility group box 1 . Pathobiology 77 : 210-217. Link: http://bit.ly/2XbXBGI

15. Li W, Hu ZF, Chen B, Ni GX (2013) Response of C2C12 myoblasts to hypoxia: the relative roles of glucose and oxygen in adaptive cellular metabolism. Biomed Res Int 2013: 326346. Link: http://bit.ly/3b61Ewg

16. Grabiec K, Gajewska M, Milewska M, Blaszczyk M, Grzelkowska-Kowalczyk K (2014) The influence of high glucose and high insulin on mechanisms controlling cell cycle progression and arrest in mouse $\mathrm{C} 2 \mathrm{C} 12$ myoblasts: the comparison with IGF-I effect. J Endocrinol Invest 37: 233-245. Link: http://bit.ly/3ndJMSv

17. Warburg O, Wind F, Negelein E (1927) The Metabolism of Tumors in the Body. J Gen Physiol 8: 519-530. Link: http://bit.ly/3njvf7Q

18. Gatenby RA, Gillies RJ (2004) Why do cancers have high aerobic glycolysis? Nat Rev Cancer 4: 891-899. Link: http://bit.ly/3pNOidW

19. Boison D (2017) New insights into the mechanisms of the ketogenic diet. Curr Opin Neurol 30: 187-192. Link: http://bit.ly/3bcnRZw 
20. Dugdale HF, Hughes DC, Allan R, Deane CS, Coxon CR, et al. (2018) The role of resveratrol on skeletal muscle cell differentiation and myotube hypertrophy during glucose restriction. Mol Cell Biochem 444: 109-123. Link: http://bit.ly/3rW3Zje

21. Mori T, Ohmori H, Luo Y, Mori S, Miyagawa Y, et al. (2019) Giving combined medium-chain fatty acids and glucose protects against cancerassociated skeletal muscle atrophy. Cancer Sci 110: 3391-3399. Link: http://bit.ly/3rUorBb

22. McCarty MF, DiNicolantonio JJ (2016) Lauric acid-rich medium-chain triglycerides can substitute for other oils in cooking applications and may have limited pathogenicity. Open Heart 3: e000467. Link: http://bit.ly/2L33rYb

23. Fauser JK, Matthews GM, Cummins AG, Howarth GS (2013) Induction of apoptosis by the medium-chain length fatty acid lauric acid in colon cancer cells due to induction of oxidative stress. Chemotherapy 59: 214-224. Link: http://bit.ly/3ojRMD0

24. Kadochi Y, Mori S, Fujiwara-Tani R, Luo Y, Nishiguchi Y, et al. (2017) Remodeling of energy metabolism by a ketone body and medium-chain fatty acid suppressed the proliferation of CT26 mouse colon cancer cells. Oncol Lett 14: 673-680. Link: http://bit.ly/390I1D1

25. Glatz JFC, Luiken J JFP (2018) Dynamic role of the transmembrane glycoprotein CD36 (SR-B2) in cellular fatty acid uptake and utilization. J Lipid Res 59: 1084-1093. Link: https://bit.ly/3rUj19d

26. Knottnerus SJG, Bleeker JC, Wüst RCI, Ferdinandusse S, IJlst L, et al. (2018)
Disorders of mitochondrial long-chain fatty acid oxidation and the carnitine shuttle. Rev Endocr Metab Disord 29: 93-106. Link: http://bit.ly/38eFo1x

27. Metges CC, Wolfram G (1991) Medium- and long-chain triglycerides labeled with ${ }^{13} \mathrm{C}$ : a comparison of oxidation after oral or parenteral administration in humans. J Nutr 121: 31-36. Link: http://bit.ly/3hKBvnP

28. Manzo E, O'Conner AG, Barrows JM, SD D, Birchak GJ, et al. (2018) MediumChain Fatty Acids, Beta-Hydroxybutyric Acid and Genetic Modulation of the Carnitine Shuttle Are Protective in a Drosophila Model of ALS Based on TDP43. Front Mol Neurosci 11: 182. Link: http://bit.ly/3rWPIrU

29. Caudron-Herger M, Diederichs $S$ (2018) Mitochondrial mutations in human cancer: Curation of translation. RNA Biol 15: 62-69. Link: http://bit.ly/2JIA50C

30. Lee YK, Jee BA, Kwon SM, Yoon YS, Xu WG, Wang HJ, et al. (2015) Identification of a mitochondrial defect gene signature reveals NUPR1 as a key regulator of liver cancer progression. Hepatology 62: 1174-1189. Link: http://bit.ly/35rJYaX

31. Montgomery MK, Osborne B, Brandon AE, O'Reilly L, Fiveash CE, et al. (2019) Regulation of mitochondrial metabolism in murine skeletal muscle by the medium-chain fatty acid receptor Gpr84. FASEB J 33: 12264-12276. Link: http://bit.ly/3940zCj

32. Miyagawa Y, Mori T, Goto K, Kawahara I, Fujiwara-Tani R, et al. (2018) Intake of medium-chain fatty acids induces myocardial oxidative stress and atrophy. Lipids Health Dis 17: 258. Link: http://bit.ly/38h7YPP
Discover a bigger Impact and Visibility of your article publication with Peertechz Publications 